\title{
Trabajo colaborativo: una experiencia desde el aula de pre grado
}

Mg. Luis Vásquez Paulini

\begin{abstract}
"El aprendizaje colaborativo se basa en el planteamiento de actividades en las que los alumnos tienen que trabajar en equipo para conseguir la interdependencia positiva"
\end{abstract}

\section{RESUMEN}

En este artículo se reporta las experiencias desde las aulas de pregrado modalidades A y B de los últimos ocho años, con el objetivo de mostrar la eficacia del trabajo colaborativo como estrategia didáctica grupal propiciadora del aprendizaje y desarrollo de destrezas. Las experiencias se desarrollaron y siguen aplicándose en las diferentes asignaturas tenidas a cargo en las aulas de pregrado en la Facultad de Educación y Psicología de la Universidad Marcelino Champagnat de Surco-Lima Perú. Nuestro relato de las mismas es sólo uno más de los múltiples relatos posibles en el camino de construir actividades didácticas, que nos permitan desarrollar destrezas, interdependencia positiva y a la vez el aprendizaje de los contenidos. Concluimos con el deseo de que otros docentes de la universidad compartan sus experiencias de investigación y docencia en el aula, que nos permitan construir nuestro recorrido teórico-metodológico particular en las aulas de pre grado.

Palabras clave: trabajo colaborativo, destrezas, actividades didácticas, interdependencia positiva.

\section{SUMMARY}

This article reports the experiences from the A and B undergraduate classes of the last eight years, with the aim of showing the effectiveness of collaborative work as a group didactic strategy that promotes learning and skill development. The experiences were developed and continue to be 
Mg. Luis Vásquez Paulini

applied in the different subjects held in the Pre-Grade classrooms at the Faculty of Education and Psychology of the Marcelino Champagnat University of Surco-Lima Peru. Our account of them is just one of the many possible stories in the way of constructing didactic activities, which allow us to develop skills, positive interdependence and at the same time learning the contents. We conclude with the wish that other teachers of the university share their experiences of research and teaching in the classroom, which allow us to build our particular theoretical-methodological journey in the pre-grade classrooms.

Key words: collaborative work, skills, didactic activities, posterity interdependence.

... Ser profesor significa saber cambiar la situación presente de tal manera que otra persona se haga mejor o como decía el padre Champagnat buen cristiano y honrado ciudadano...

\section{INTRODUCCIÓN}

En educación es conocido que el aprendizaje-enseñanza de ciencias o cualquier asignatura de ella, lleva a los estudiantes a reflexionar, experimentar, inferir, intuir, deducir, prever, memorizar, escuchar, ver, observar, trabajar en grupo, en pares o individualmente. Es decir desarrollar o continuar desarrollando las destrezas que se necesitan para utilizar adecuadamente la metodología científica. Para los profesores de ciencias no es novedad y nada difícil elaborar actividades para el trabajo individual, en pares y en grupos, puesto que el trabajo diario en el laboratorio así lo amerita. El reto era trasladar esta experiencia a las aulas, en actividades diarias usando los contenidos como medios para continuar desarrollando las destrezas de acuerdo a la propuesta de la Universidad y ni qué decir de la evaluación que en ese entonces era un punto que estaba en debate, en discusión y porque no decirlo se consideraba un punto caliente.

La elaboración de las lecturas para las actividades diarias, nos llevó años en su dosificación y actualización, en ellas se plasman los contenidos de acuerdo al currículo de la universidad. Hoy contamos con 30 lecturas para cada asignatura, las mismas que actualizamos cada dos años o si el currículo de la facultad cambia. Cada año se informaba como logro al Decanato.

A continuación se incluye la lectura para la actividad 1 de la asignatura Biología Humana I. Agosto 2017. 
Universidad Marcelino Champagnat

Profesor: Mg. Luis

Vásquez Paulini

Bio-elementos y Bio-moléculas

Unidad: 1

\section{LECTURA-TALLER -LABORATORIO 2}

Biomoleculas-El Agua

\section{Actividad No 1 La historia del Agua}

Elaborar conclusiones sobre el contenido de la lectura la historia del Agua, a través de la elaboración de una infografía que permita realizar inferencias adecuadas de su importancia en la vida tal como la conocemos. Trabaja en pares y grupos colaborativamente aceptando distintos puntos de vista.

\section{Lee el contenido de la lectura la historia del Agua.}

2. Identifica y selecciona los más relevantes.

3. Contextualiza la situación y las compara.

\section{Establece relación causa efecto.}

\section{Elabora-obtén conclusiones.}

Hace 4000 millones de años (4 billones) parte del agua que hay en el planeta llego desde el espacio exterior donde enormes cantidades de hidrógeno y oxígeno se unieron, para formar lo que conocemos como hielo espacial, los cometas promedio, están formados por más de un millón de toneladas de hielo, los humanos y todas las formas de vida debemos parte de nuestra existencia a esta agua extraterrestre. La otra fuente de la cual proviene el agua de nuestro mundo es de la combinación de oxigeno e hidrógeno en las profundidades del planeta, que se manifiesta en forma de vapor de agua hirviendo que emerge a manera de geiseres o manantiales volcánicos; en los 3 000 millones de años siguientes la atmosfera se saturo de vapor de agua, esta capa de vapor que rodeaba a la tierra la protegía de los abrazadores rayos del sol. También atrapaba el calor solar y con el paso del tiempo la atmosfera de la tierra comenzó a calentarse, de no ser por este primer efecto invernadero, la temperatura media de la tierra seguiría siendo de $-18^{\circ} \mathrm{Centigrados}$ y nuestro mundo sería un desierto helado. La vida tal como la conocemos no podría haber prosperado. Con el tiempo la atmosfera comenzó a calentarse lo suficiente para que empezase a caer lluvia, el agua de la lluvia lleno los océanos y la energía del sol empezó a impulsar el ciclo del agua a través de todo el planeta un ciclo que perdura hoy en día. Cada día que pasa se pierde agua a través de la parte superior de la atmosfera. ¿Agotaremos algún día nuestra reserva de agua? En el centro de la tierra, a treinta kilómetros por debajo de la corteza terrestre las temperaturas sobrepasan los 1000 grados centígrados. En el nivel superior del manto (capa que separa la corteza del núcleo) lugar colmado de rocas calientes, pero en algunas partes más profunda también hay agua, posiblemente halla 10 veces los volúmenes de todos los océanos del mundo combinados, este colosal embalse se encarga de reemplazar el agua que se pierde en el espacio exterior, se libera como vapor cada vez que algún volcán erupciona. Los volcanes se encargaron de dar forma al planeta en un inicio, 
pero estaban debajo del agua y allí liberaban todo su vapor directamente al agua. Los océanos reciben agua de grietas de los abismos (fumarolas negras) que expulsan minerales vaporizados y agua a altísimas temperaturas.

\section{Trabajo personal}

- Lee el texto el agua y sus propiedades. Resalta las ideas principales, subraya las ideas secundarias y elabora un organizador grafico de tu elección.

- Metacognición: ¿Qué sabías antes de la lectura? ¿Qué aprendiste después de la lectura? ¿Cómo lo aplicarías en tu vida practica?

\section{Trabajo por pares}

- Compartan y expliquen sus organizadores gráficos.

\section{Trabajo colaborativo}

- Diseñen una infografía que sea útil para graficar la historia del agua.

\section{Indagación. Participar en el foro y clase.}

- ¿Qué sucede en nuestro cuerpo con el agua que nos tomamos?

Fuente:

- Tortora, G. y Derrickson, B. (2013). Principios de Anatomía y Fisiología. 13a Edición. México: Panamericana. 
Solucionado el problema de las lecturas-contenidos. Nuestro segundo reto fue investigar la metodología del trabajo en grupo en las aulas. Les comento que me ayudo mucho las investigaciones que realice sobre el estado del arte, para mi proyecto de tesis... "Estrategias de Aprendizaje Colaborativo en el desarrollo de actitudes ambientales en alumnos de la asignatura de Educación Ambiental de la Facultad de Educación y Psicología de la Universidad Marcelino Champagnat" Que luego se convirtió en tesis para obtener el grado de Magister.

\section{EXPERIENCIA DESDE EL AULA DE PRE GRADO}

Una y otra vez en diferentes tesis, tesinas, investigaciones, proyectos, sobre el trabajo en grupo investigados y leídas, se repetían de manera constante los apellidos de dos investigadores Bruffee(1), Johnson, D. y Johnson, R. A los cuales me remitiré textualmente en este párrafo y les comentare la discrepancia que tengo con ellos al haberles desarrollado en la práctica, la metodología y teoría sugerida en cuanto al trabajo en grupo, en estos últimos años y en el 2017 que está por finalizar.

La metodología (modificada) que se utiliza en las aulas de pre-grado y que desarrollaremos más adelante, es semejante al modelo que Bruffee (1) llama consensus-group collaborative learning (cap.2), a lo descrito por Johnson, D., Johnson, R. (2). Los nuevos círculos del aprendizaje y a la propuesta de la Universidad Marcelino Champagnat (3). Marino Latorre Ariño y Carlos Javier Seco del Pozo Diseño curricular nuevo para una nueva sociedad, según el Paradigma Socio-cognitivohumanista, Modelo T.

Se le puede resumir en estos seis pasos:

- Consenso a través de la colaboración en el aprendizaje.

- Participación voluntaria en el proceso.

- Aprendizaje no fundacional: se trabajan preguntas con respuestas debatibles, que no son únicas.

- Cambio en la relación profesor-estudiante: la autoridad pasa del profesor a grupos de pares y luego a equipos colaborativos.

- Se discuten los contenidos.

- Importancia del trabajo individual y diálogo entre pares.

Discrepamos con Bruffee (1), con Johnson, D. W., and Johnson, R. T. () y Marino Latorre Ariño y Carlos Javier Seco del Pozo (3) en el número de integrantes por grupo y forma de integrarse. Asumimos un número de tres integrantes por grupo, se unen por afinidad y cada uno cumple y se posesiona de un rol rotativo al interior del mismo (Expositora-Secretaria-Coordinadora de materiales).

Los grupos colaborativos requieren tareas-actividades donde el conocimiento se construye con medios no siempre conocidos. No suponen una única respuesta ni un único método para llegar a ella. Dichas tareas llevan a los estudiantes a un proceso de conversación espontánea y constructiva. 
Adaptan su propia terminología a los nuevos propósitos. Se trata de un constructo, al que llegan los estudiantes con sus propios medios.

En consecuencia el trabajo colaborativo lo podemos definir como los aportes que hace un integrante del grupo a sus compañeros de equipo en cuanto a experiencias, comentarios, sugerencias y reflexiones sobre el trabajo que se ha desarrollado, y a su vez, espera que sus compañeros de equipo contribuyan en el mismo sentido. Para después transformar el trabajo individual en un producto más rico. El trabajo en equipo es una de las características del trabajo colaborativo que se manifiesta cuando aprenden juntos a resolver problemas, habilidades, liderazgo, comunicación, confianza, toma de decisiones.

La autoevaluación, coevaluación y metacognición periódicamente identifican debilidades a mejorar en el futuro.

La comunicación: intercambiar información efectiva y eficiente, mejora en claridad las conclusiones.

La responsabilidad: comprende trabajo individual de cada uno de los integrantes, el trabajo en pares y el trabajo colaborativo. La responsabilidad está ligada a la noción de reciprocidad.

Sobre la Interdependencia positiva. La responsabilidad grupal en el trabajo colaborativo se le conoce en la literatura como interdependencia positiva. Esta se centra en la noción de que ninguno de los miembros, individualmente, podrá alcanzar el éxito si todos los demás no lo alcanzan. La interdependencia positiva es central en el trabajo colaborativo por cuanto facilita la organización y funcionamiento del grupo.

Se hace indispensable, tanto para los profesores como para los alumnos, comprender que la actividad colaborativa sólo se logrará cuando es asumida conscientemente en la interrelación docente y estudiante. El cierre de la clase o tema si lo amerita lo realiza el profesor. (Ver foto 1 Trabajo colaborativo).

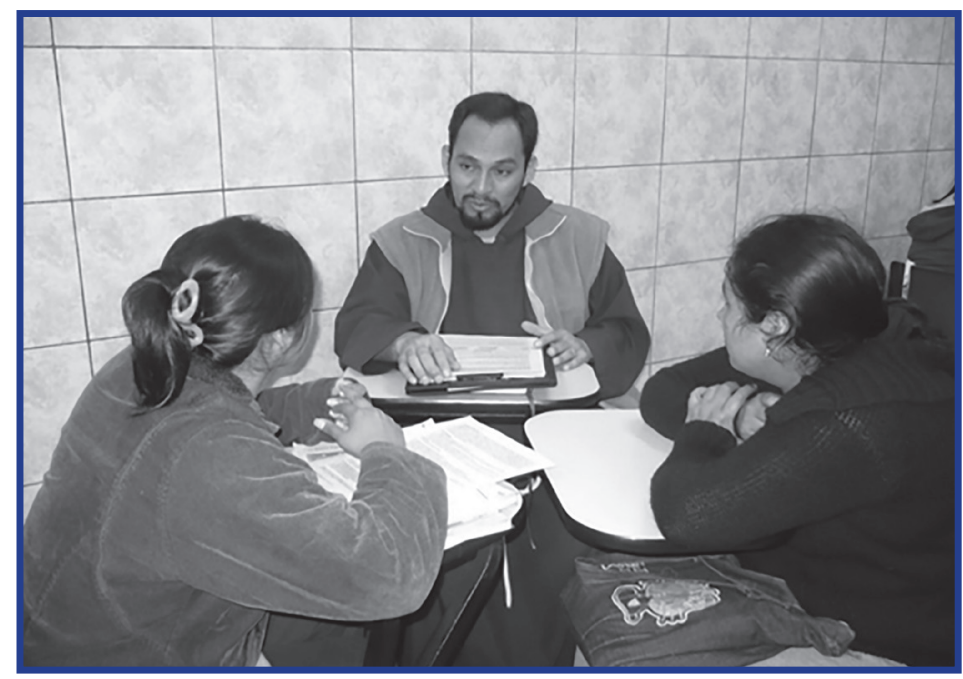

Foto 1. Trabajo Colaborativo 


\section{CUADERNO COLABORATIVO Y DISPOSICIÓN DE LAS CARPETAS}

En lo que se refiere al trabajo en grupo colaborativo, se tuvo que idear una caratula para un cuaderno, al que llamamos cuaderno colaborativo, en donde por consenso informan los resultados de la actividad realizada y que se socializa a la clase u otro grupo colaborativo.

El cuaderno colaborativo consta de una caratula donde se colocan el número de grupo, sección y nombre de sus integrantes (3-elegidos por afinidad). Los integrantes del grupo colaborativo se comprometen a asumir cada uno un rol, el mismo que rotara en cada actividad. Su trabajo tiene como objetivo lograr la interdependencia positiva, es decir que todos deben saber todo de la actividad, asumir el liderazgo y socialización de sus conclusiones.

Existe un área en donde deben colocar una norma o compromiso a tener en cuenta durante las actividades (contrato didáctico).

En las caras siguientes de la caratula se han colocado las definiciones de las destrezas a desarrollar durante el ciclo y su pasos mentales de acuerda a la propuesta de la universidad.

Por otro lado se establecen también áreas de autoevaluación, coevaluación y metacognición.

La caratula de cuaderno colaborativo permite recordar los roles, la destreza que se está desarrollando y el compromiso a tener en cuenta en la actividad. Asimismo los integrantes del grupo acceden a la autoevaluación, la coevaluación y la metacognición. (Ver fotos 2 y 3 del cuaderno colaborativo)

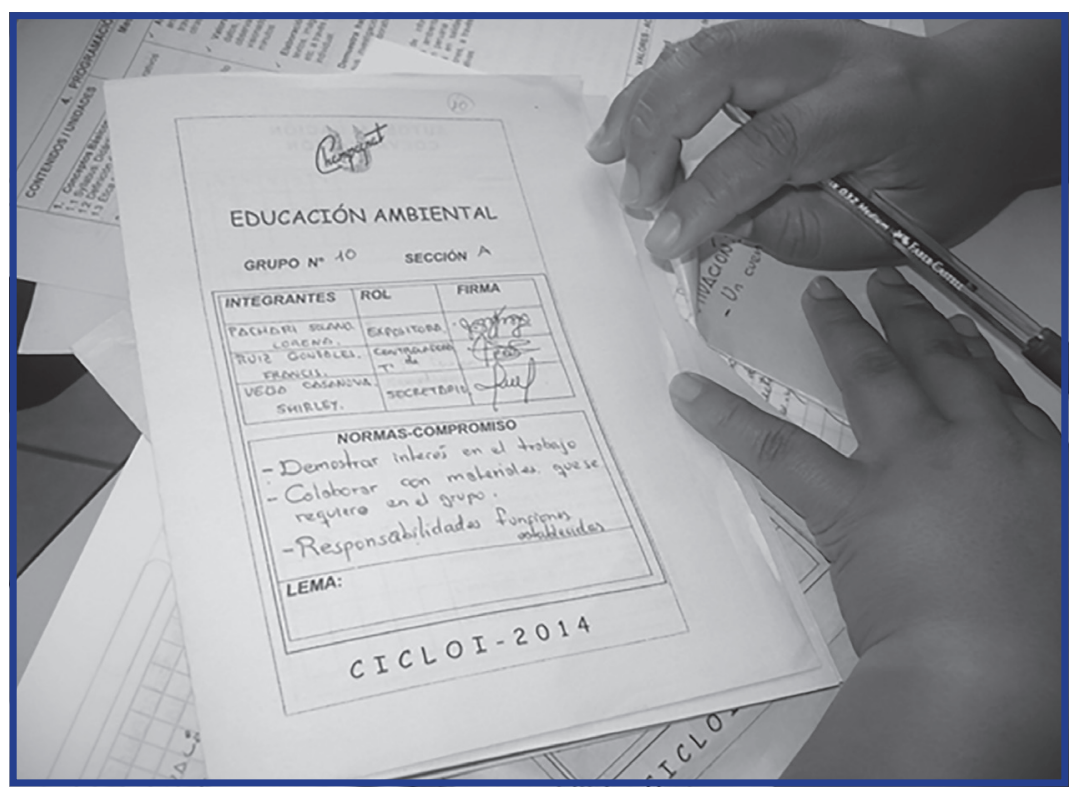

Foto 2. Caratula del cuaderno colaborativo. 
Mg. Luis Vásquez Paulini

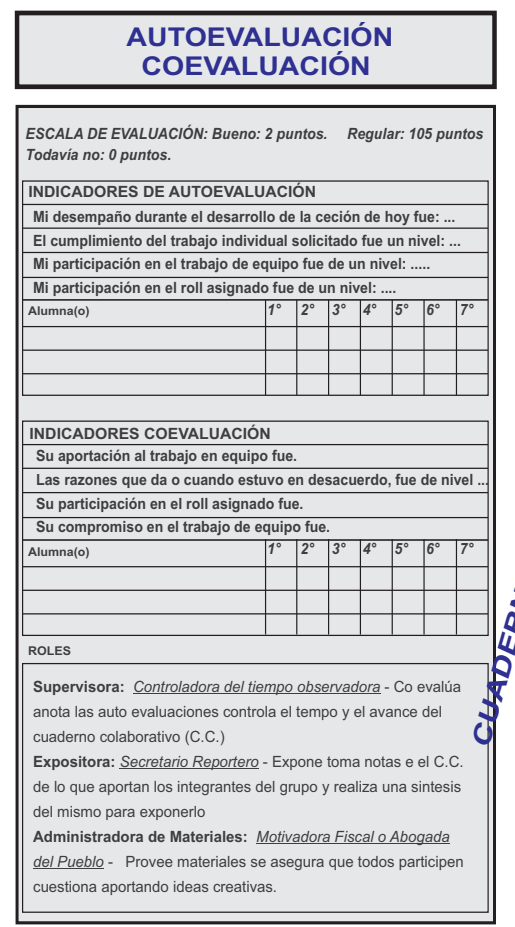

\begin{tabular}{|c|c|c|}
\hline \multicolumn{3}{|c|}{$\begin{array}{c}\text { DESTREZAS } \\
\text { PROCESOS MENTALES }\end{array}$} \\
\hline 1 & $\begin{array}{l}\text { IDENTIFICAR } \\
\text { Habilidad especifica para } \\
\text { reconocer las caracteristicas } \\
\text { esenciales de objetos, hechos } \\
\text { fenómeno, personajes, etc, que } \\
\text { hacen que sea lo que son. }\end{array}$ & $\begin{array}{l}\text { Percibir la información de forma } \\
\text { clara. } \\
\text { Reconocer las caracterisiticas } \\
\text { relacionar (comparar) con los } \\
\text { conocimientos previos que se } \\
\text { tienen sobre el objeto. } \\
\text { Identificar }\end{array}$ \\
\hline 2 & $\begin{array}{l}\text { ANALIZAR } \\
\text { Habilidad especifica para esperar } \\
\text { las partes esenciales de un todo, } \\
\text { a fin de llegar a conocer sus } \\
\text { principios y elementos entre las } \\
\text { partes que forman un todo. }\end{array}$ & $\begin{array}{l}\text { Percibir la información de forma } \\
\text { clara. } \\
\text { Identificar las partes esenciales } \\
\text { Relacionar las partes entre si } \\
\text { Realizar analisis }\end{array}$ \\
\hline 3 & $\begin{array}{l}\text { INTERPRETAR } \\
\text { Habilidad especifica para atribuir } \\
\text { significado o sentido a } \\
\text { determinada información, sea } \\
\text { texto, dibujos, signos, símbolos } \\
\text { huellas, especificas artisiticas }\end{array}$ & $\begin{array}{l}\text { Percibir la información de forma } \\
\text { clara. } \\
\text { Decodificar lo percibido(dibujos, } \\
\text { signos, texto). } \\
\text { Relacionar con experiencias y } \\
\text { saberes previos } \\
\text { Agregar significado o sentido }\end{array}$ \\
\hline 4 & $\begin{array}{l}\text { DEFINIR } \\
\text { Habilidad especifica para } \\
\text { expresar en términos breves el } \\
\text { significado de un concepto, } \\
\text { objeto, fenómeno o situación }\end{array}$ & $\begin{array}{l}\text { Conocer y comprender el concepto } \\
\text { que se quiere definir. } \\
\text { Determinar sus carateristicas } \\
\text { esenciales. } \\
\text { Expresar las caracterisitcas. } \\
\text { Definir }\end{array}$ \\
\hline 5 & $\begin{array}{l}\text { SINTETIZAR } \\
\text { Habilidad especifica para reducir } \\
\text { a términos breves y precisos el } \\
\text { contenido esencial de una } \\
\text { información }\end{array}$ & $\begin{array}{l}\text { Analizar } \\
\text { Sintetizar mediante un organizador } \\
\text { gráfico o elaborando un texto } \\
\text { breve } \\
\text { Sintetizar } \\
\end{array}$ \\
\hline 6 & $\begin{array}{l}\text { APLICAR } \\
\text { Habilidad especifica para utilizar } \\
\text { procedimiento, técnica, leyes o } \\
\text { herramientas, etec, para explicar, } \\
\text { realizar o solucionar una } \\
\text { situación problematica }\end{array}$ & $\begin{array}{l}\text { Percibir la información de } \\
\text { forma clara. } \\
\text { Identificar ley o principio } \\
\text { herramienta que se va a utilizar } \\
\text { Utilizar ley de principio } \\
\text { Aplicarlo }\end{array}$ \\
\hline
\end{tabular}

Foto 3. Evaluación, roles y destrezas en el cuaderno colaborativo.

Sobre la disposición de las carpetas, se colocarán dos con el tablero uno al frente del otro y el tercer tablero en medio de los dos. Esto permite por prevención si hay un sismo, a los alumnos salir sin que se tropiecen los unos con los otros. (Ver fotos 4 y 5 disposición de las carpetas y trabajo personal).

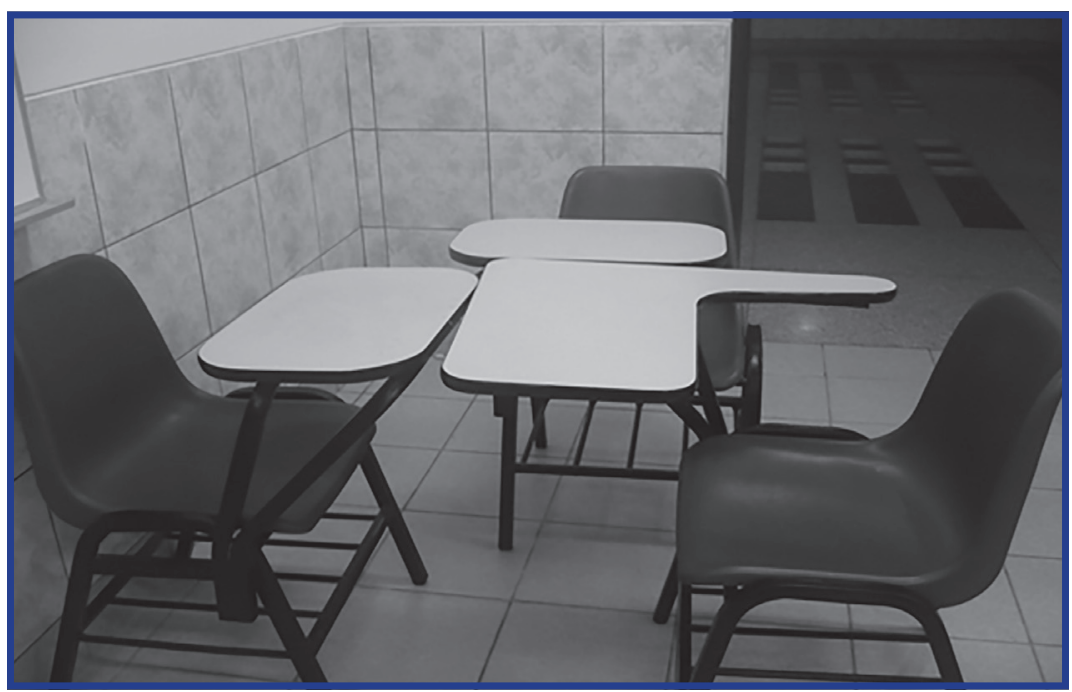

Foto 3. Disposición de las carpetas 


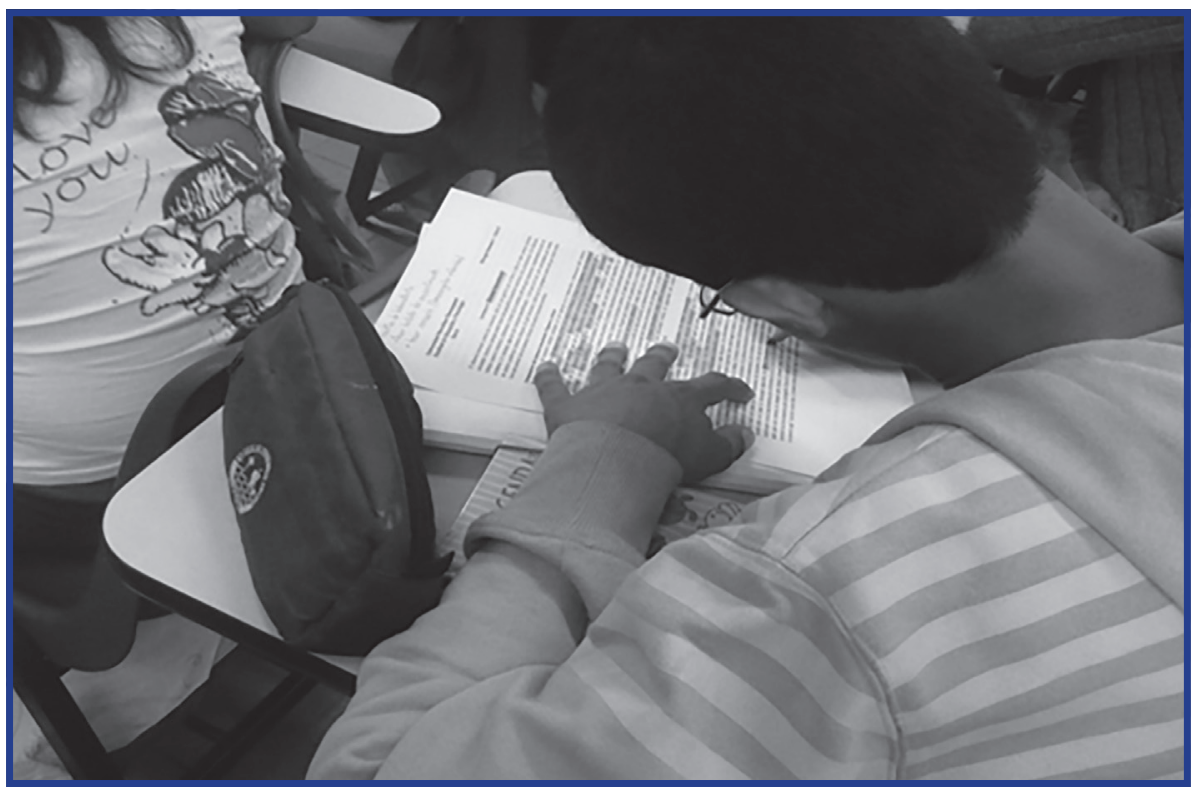

Foto 4. Trabajo personal

\section{LAS HORAS DE CLASES}

La famosa ultima hora (mañana y tarde). Imagino que todos hemos tenido la experiencia de la ultima hora en Instituciones educativas, Institutos y Universidades, donde hemos trabajado.

Recuerdo que a pesar del agotamiento, fruto del transcurrir del día, llegaba la famosa última hora, no está demás decir que, como estamos al tanto, la mayoría de nuestros alumnos trabajan por la mañana, viven lejos de la Universidad y almuerzan en el comedor de la universidad o frente al espejo de agua del humedal artificial.

Se tuvo que idear actividades específicas para esta hora. Se iniciaba la actividad con el trabajo por pares; no con el trabajo individual. En donde el tema a tratar se conversaba y luego se mejoraba colaborativamente utilizando la lectura del trabajo personal; paradójicamente el tiempo de la clase pasaba volando y lograban asimilar los contenidos incluidos en la lectura. Creo que, en aquellas circunstancias, hacerles aprender a aprender, fue uno de los muchos logros obtenidos al aplicar el trabajo colaborativo como estrategia personal. (Ver fotos pág. 209)

Me gustaría escribir aquí un ejemplo de como he usado la estrategia en clase la ultima hora, estos últimos ocho años, estrategia que he adaptado para las clases de las diferentes asignaturas que imparto en la Universidad para la famosa ultima hora.

Respecto a lo que se comenta sobre el juego y las canciones en la literatura educativa, al utilizarlas para el desarrollo de actividades en clase y su aprendizaje; es que el juego y las canciones son actividades específicas para los niños y adolescentes, pero no para los adultos y que su uso debe 
Mg. Luis Vásquez Paulini

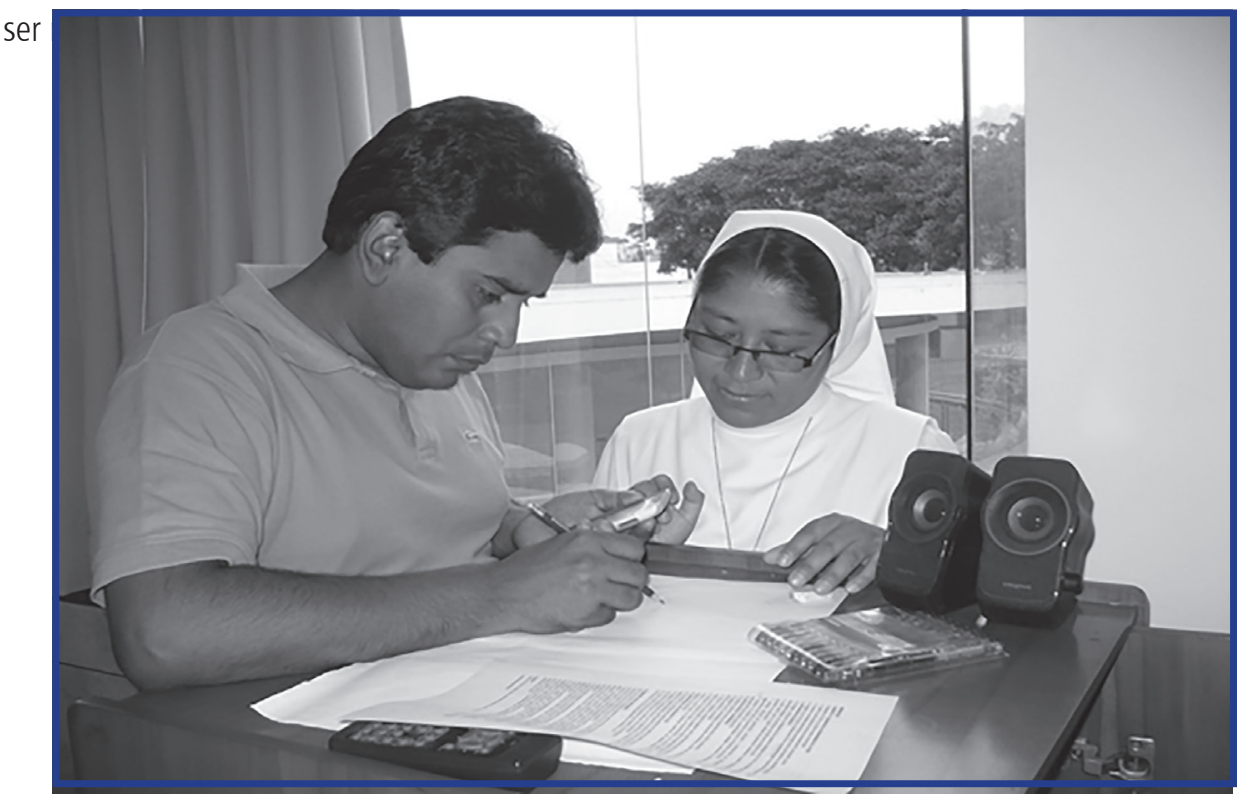

Foto 5. Trabajo en pares

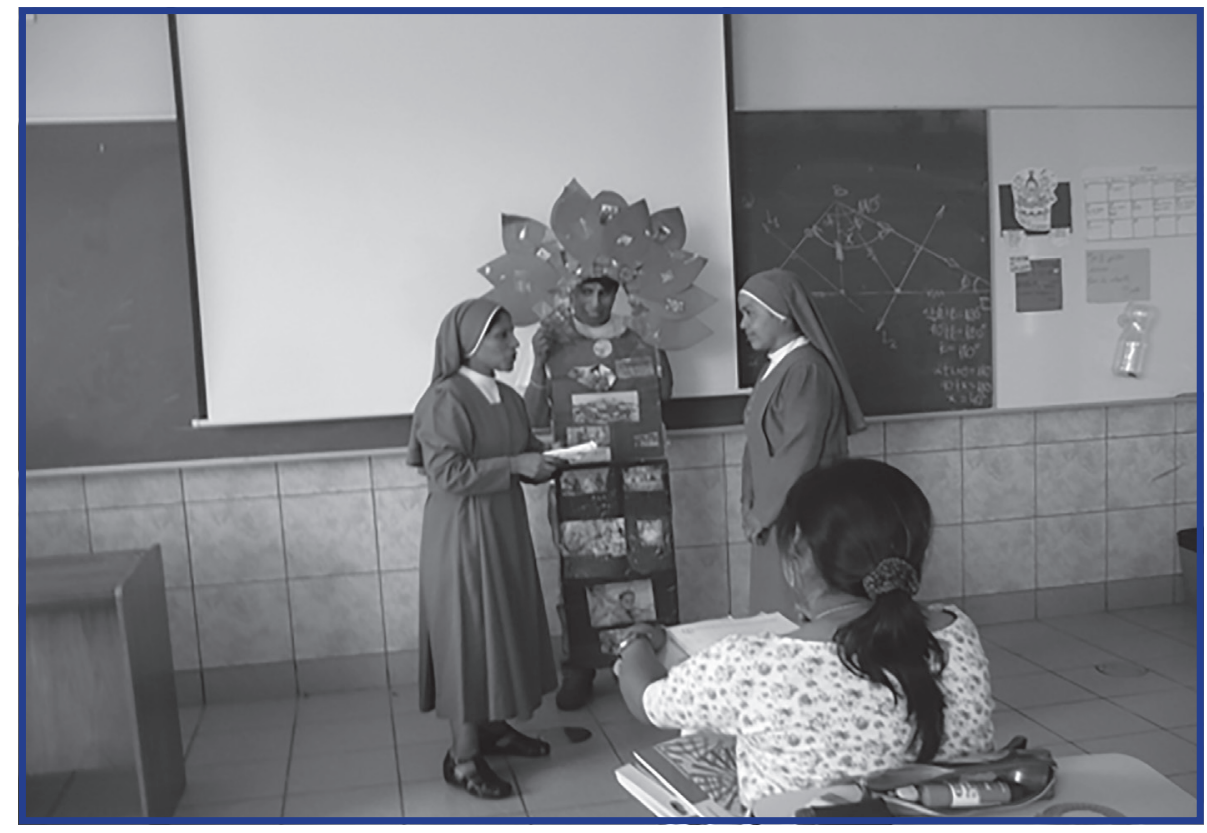

Foto 6. Socialización de lo aprendido 
La experiencia me ha enseñado que el juego y las canciones son importantes para la vida de los niños y también los adultos. La historia de la humanidad nos demuestra que los humanos siempre hemos jugado y cantado. El juego y el canto de acuerdo a las investigaciones realizadas en el Escuela Experimental № 7011-Uladislao Zegarra Araujo de Miraflores-Perú, referente al Método Peruano de Lectura y Escritura, autores Desiderio Vásquez Aguilar y Juan Vásquez Vigo, entre los años 40-70, lo definen como una forma de comunicación y que lo desarrollamos desde pequeños, cuando queremos contactar con los adultos. Siendo útil para aprender. (Proyecto de mi tesis doctoral "Evaluación de los resultados del Método Peruano de Lectura y Escritura en la Escuela Experimental No 7011-Uladislao Zegarra Araujo de Lima, a partir de los avances actuales de las Ciencias Pedagógicas" UMCh-2013- Asesor: Dr. Ángel Velásquez).

Acercándonos a este terreno particular... Para elaborar actividades lúdicas y/o experiencias gamificadas, utilizaba en clase los juegos del rol, viajes imaginarios, "programas de noticias", cuentos científicos, canciones de moda (letra y melodía), contranuncios, dilemas morales y los juegos de su Smartphone, etc., tratando de conectar constantemente la lectura de su trabajo personal cuya duración es de 10 minutos con el uso de lo que he llamado el cuaderno colaborativo, en donde diseñan la estrategia-juego-canto con el que participaran en la socialización de sus conclusiones. Lo más relevante de todo estos, es que a través del juego y las canciones aprendemos a estar juntos, a estar con los demás, a negociar, a dialogar. Es crucial. Aprendes a interpretar lo que piensa el otro, a ceder, a defender lo que crees. También aprendes a colaborar, pones en práctica la democracia. Los participantes han de decidir a qué juegan-cantan, quién encarna cada rol, cómo cambian estos roles a lo largo del juego-canto... Se pasan el rato hablando, negociando. Y como uno de los objetivos del juego-canto es que dure en el tiempo, suelen ponerse de acuerdo. Incluso hay juegos-canciones, como el de tocar y parar, en el que se discutirán las normas -hasta dónde se puede correr, cómo te salvas..., y si alguien las vulnera debe debatirse cómo se actúa... ¿Qué te parece?... Las propuestas de actividades donde incluyas la gamificación (ludificación) son interesantes y válidas.

\section{NO OLVIDEMOS LA MOTIVACIÓN}

La motivación. En estos momentos de reflexión, me viene a la memoria la conversación en pasillos, que tuvimos, saliendo del aula azul con el docente Iván Giraldo y el Hno. Marino Latorre, sobre el significado de «modernidad líquida» del sociólogo Zygmunt Bauman (2008), término que escuché en una mesa del I Seminario Internacional: Investigación Científica en la Universidad, realizada en el mes de octubre del presente año. En el citado evento participó como panelista el Dr. Ángel Velásquez (asesor de tesis). A quienes les comentaba que el concepto de Modernidad liquida se me había quedado a medias y les preguntaba sobre si tenían algunas copias sobre ella... Luego ellos, la definieron "como el estado fluido y volátil de la actual sociedad, sin valores demasiado sólidos, en la que la incertidumbre por la vertiginosa rapidez de los cambios ha debilitado los vínculos humanos". Luego los dos comentaron al unísono, sobre mi deseo de aprender y salió el término motivación intrínseca... Que podemos definirla "como la que nos impulsa a hacer cosas por el simple gusto de hacerlas. La propia ejecución de la tarea es la recompensa". 
Por consiguiente, nadie aprende algo solo por aprenderlo, lo aprende porque lo quiere como un instrumento de comunicación, lo aprende para usarlo; en el trabajo por pares y en el trabajo en grupos colaborativos, las actividades elaboradas motivan a nuestros alumnos el aprender los contenidos (trabajo personal) y luego le enseñamos a usarlo, a aplicarlo a situaciones concretas (trabajo por pares y colaborativos) y estar preparados para otra realidad social y cultural futura.

\section{LA EVALUACIÓN}

Volviendo a la evaluación. La resistencia que hemos encontrado por parte de los estudiantes, para corregir los trabajos de sus pares, parece ser común al empleo de esta estrategia pedagógica. No encontramos una mejor descripción que la cita, aunque extensa, de Bruffee al respecto: "Cualquier profesor que solicite a sus estudiantes criticar [corregir] el trabajo de sus compañeros, hallará fuerte resistencia; sin embargo, le satisfará saber que la mayoría vencen dicha resistencia. La reacción inicial de cualquier estudiante que se le pida corregir el trabajo de su compañero(a) lo interpretará como un juego sucio hecho al amigo(a): la crítica mutua como un modo de traición. Si el profesor logra vencer ese prejuicio, el comentario hecho al otro será de total complacencia, o su extremo opuesto: una crítica descarnada y desalentadora. Al principio el estudiante afirma no encontrar nada errado en el trabajo de su compañero(a); luego, afirma que no hay nada valioso. Pasan de ser complacientes a ser unas pirañas. Ambas reacciones son típicas de solidaridad de grupo, donde unos se apoyan con lealtad, pero cierran filas contra otros a quienes excluyen. Ninguna de estas acciones desarrolla la interacción e interdependencia positiva que contribuye a juicios maduros.

La estrategia del aprendizaje colaborativo supone que los conflictos en relación con la autoridad del profesor y los cuestionamientos a los puntos de vista de los otros estudiantes del grupo, y la resistencia a ser evaluado o evaluar a sus pares, son aspectos inevitables y necesarios del proceso de aprendizaje.

\section{CONCLUSIÓN}

Los resultados de la eficacia del trabajo colaborativo, trabajo por pares y trabajo individual; para desarrollar las destrezas y el aprendizaje de contenidos, así como los materiales elaborados, han revelado un aprovechamiento sustancial de las actividades propuestas, que se reflejan no sólo en la calidad de los conocimientos adquiridos por los estudiantes, sino también en el reconocimiento que los propios estudiantes hacen del aprendizaje alcanzado en la dimensión personal-social (destrezas) y las relaciones profesor-alumno. Estas experiencias representan a mi modo de ver prácticas educativas útiles para la mejora del aprendizaje en la universidad.

En este contexto concluimos con el deseo de que otros docentes de la universidad compartan sus experiencias de investigación y docencia en el aula, que nos permitan consolidar nuestro recorrido teórico-metodológico particular en las aulas de Pre Grado. 


\section{REFERENCIAS}

1. Bruffee, K. (1999). Collaborative Learning, Higher Education, Interdependence and the Authority of Knowledge, Baltimore: The Johns Hopkins University Press, Second edition.

2. Johnson, D., Johnson, R.y Johnson, E. (1999). Los nuevos círculos del aprendizaje. Argentina: Aique

3. La Torre, M. y Seco, C. (2010). Diseño curricular nuevo para una nueva sociedad, según el Paradigma Socio-cognitivo-humanista, Modelo T. Perú: visionpcperu

4. Vásquez, L. (2012). Estrategias de Aprendizaje Colaborativo en el desarrollo de actitudes ambientales en alumnos de la asignatura de Educación Ambiental de la Facultad de Educación y Psicología de la Universidad Marcelino Champagnat. Perú:UNE.

5. Vásquez, L. (2013). Proyecto de tesis doctoral. Evaluación de los resultados del Método Peruano de Lectura y Escritura en la Escuela Experimental No 7011-Uladislao Zegarra Araujo de Lima, a partir de los avances actuales de las Ciencias Pedagógicas. Perú: UMCh. 